\title{
A REVIEW OF THE STATE OF THE ART IN ARTIFACT REMOVAL TECHNOLOGIES AS USED IN AN ASSISTED LIVING DOMAIN
}

\author{
K.T. Sweeney*, D. Kelly ${ }^{\dagger}$, T.E. Ward ${ }^{\dagger}$, S.F. McLoone ${ }^{\dagger}$ \\ * Electronic Engingeering Dept, NUI Maynooth, Ireland: ksweeney@eeng.nuim.ie \\ ${ }^{\dagger}$ Electronic Engingeering Dept, NUI Maynooth, Ireland
}

Keywords: Artifact Removal, Adaptive Filter, Bayesian Filtering, Blind Source Separation (BSS), Independent Component Analysis (ICA)

\begin{abstract}
There has been significant growth in the area of ubiquitous, pervasive, distributed healthcare technologies due to the increasing burden on the healthcare system and the impending demographic shift towards an aging population. The move from a hospital-centric healthcare system towards in-home health assessment is aimed to alleviate the burden on healthcare professionals, the health care system and caregivers. Advances in signal acquisition, data storage and communication channels provide for the collection of reliable and useful in-home physiological data. Artifacts, arising from environmental, experimental and physiological factors, degrade signal quality and reduce the utility of the affected part of the signal. The degrading effect of the artifacts significantly increases when data collection is moved from the clinic into the home. Advances in signal processing have brought about significant improvement in artifact removal over the last number of years. This paper reviews the most common physiological and location-indicative signals recorded in the home and documents the artifacts which occur most often. A discussion of some of the most common artifact removal techniques is then provided. An evaluation of the advantages and disadvantages of each is given with reference to the assisted living environment.
\end{abstract}

\section{Introduction}

There is an impending demographic shift towards a more aged population which will place increased pressure on the worlds healthcare systems. Accompanying this burden is the rise in the annual expenditure required to facilitate this growing need. There is therefore a desire for more of the rudimentary examinations currently performed in a hospitalcentric environment to be performed in a personal, in-home, healthcare environment. By removing the patient from the hospital the cost of care can be reduced by as much as $50 \%$ [31], while simultaneously increasing the comfort of the patient in many instances.

Monitoring patient's health in their home environment allows clinicians to perform the required examinations in the patient's natural surroundings which can provide beneficial diagnostics often not available from clinical measurements. There are many ailments that require long term monitoring and in-home monitoring allows for this extended measurement period without the stress that can often encompass a hospital visit. Accompanying this desire to measure the patient's health in an assisted living environment there is often a desire to monitor or track a patient's location. This can aid a clinician to infer a large amount of information about the patient's health, for example the patient's level of mobility. However, the increased freedom for the patient comes at the cost of reduced data quality. Patient examination in a hospital is often performed in a shielded environment promoting uncontaminated recorded data, however in the assisted living domain this is not true. This increased level of noise in the acquisition domain, along with the possibility of poor procedural setup adherence, often leads to large numbers of artifacts on the desired recording with a subsequent degradation in signal utility.

In this paper we review a number of current techniques used for physiological signal and localisation signal artifact removal, with a particular emphasis on the signals and techniques that are employed in an assisted living setting. Section 2 describes some of the physiological and location indicative signals most often recorded during testing, Section 3 gives a brief explanation of the most common artifact types detected in these signal recordings, Section 4 lists and describes a number of artifact removal techniques currently utilized in signal processing and Section 5 gives a brief discussion on the findings of the review.

\section{Measurement Techniques}

As the world of medicine has evolved over the last number of decades, the capabilities of the accompanying measurement devices has also improved. This increase in capability, accompanied with the ability to store larger quantities of data, has allowed many of the tests previously undertaken in a clinical setting to be performed in an in-home environment. The most common physiological tests performed in the assisted living environment can be separated into two broad headings, electrical and optical. 
Electrical recordings, such as Electrocardiography (ECG), Electroencephalography (EEG) and Electromyography (EMG) are used to detect the changing electrical currents produced by the human body. This electrical activity is recorded using either surface or implanted electrodes; however, the use of implanted electrodes in the assisted living environment is rare due to the complex nature of application. ECG is an electrical measurement of the activity of the heart muscle. Using the recorded signal it is possible for clinicians to infer information regarding the cardiovascular health of a patient. For example, a change in the T-wave amplitude during a recording is established as an indicator for an increased risk of arrhythmias [29]. However many ailments, such as arrhythmias, occur rarely and thus establish a requirement for long term monitoring. Assessment in the assisted living domain accommodates this requirement by allowing testing to be completed in a home environment, with little time restrictions, while adding to the comfort of the patient. EMG is the measurement used to evaluate the electrical activity in the skeletal muscles. Its application encompasses many areas including use in prosthetics, in differentiating between essential and parkinsonian tremor [1] and in the area of physiological stress [21]. EEG is a measurement of the electrical activity of the brain produced by the firing of neurons. This measurement technique is used in many areas of medicine including the detection of epilepsy. Again, as with arrhythmias, epileptic activity often occurs infrequently and thus is difficult to detect in a hospital-centric environment. In the hospital environment epileptic activity is often required to be artificially induced using a number of techniques due to the time restriction on recording time. This problem can be overcome using long term monitoring in the assisted living domain.

Optical recordings are commonly employed to determine volumetric changes in the body. Photoplethysmography (PPG) and Pulse-Oximetry (POX) are the most common forms of optical measurement technique used in an assisted living environment. PPG is a non-invasive volumetric measurement of an organ. It is an inexpensive method often used to detect the cardiac cycle and respiration. POX utilizes the PPG waveform to determine the oxygenation saturation levels in the blood. This is often used in situations where a patients oxygenation levels need to be monitored such as in intensive care, as well as aiding in the diagnosis of some sleep disorders such as apnea [6].

In the field of in-home location tracking (or localisation), the aim is to translate sensor perturbations within the home environment into user location estimates. A variety of sensors can be employed, leading to two distinct forms of in-home localisation; namely those which use binary measurement sensors and those which use continuous measurement sensors. Binary measurement localisation systems can be further segmented into those which use physical interaction with switches within the environment, such as pressure mats and reed switches, and those which use remote interaction, such as Passive Infra Red (PIR) sensors [25] and the detection of Radio-Frequency (RF) signals emanating from the user [23]. $\mathrm{RF}$ localisation is particularly useful in a home physiological monitoring scenario, since the user may already be wearing some RF signal emitting devices. In such a scenario, RF localisation is preferable to PIR localisation since it is less susceptible to multiple occupancy interference, or interference from other environment inhabitants.

Continuous measurement sensors refer to sensors which produce a high degree of variability as a subject moves throughout the home environment. The aforementioned binary measurement RF localisation techniques are only relevant when the employed RF communication hardware merely produces binary detected/not-detected readings. When more detailed RF signal quality indicators are available, higher resolution location predictions can be produced. This is generally achieved by translating location-dependent signal quality indicators such as Received Signal Strength Indicator (RSSI) and Link Quality Indicator (LQI) readings into location estimates using a variety of techniques, such as triangulation or fingerprinting (see [24] for more information). Hence, the reliability of these techniques is related to the fidelity of the RSSI or LQI readings and low fidelity readings must be compensated for by using artifact removal techniques.

\section{Artifacts}

The presence of artifacts seriously degrades the clinical utility of measurements taken in a home environment. There are three distinct sources of noise that impact all signal recordings; environmental noise, experimental errors and systemic physiological artifacts [19]. These three sources have different properties and thus manifest themselves distinctively on all the separate desired signals discussed in Section 2.

\subsection{Environmental}

Environmental noise is defined as noise that contaminates the desired signal that originates from other sources external to the area being monitored. For physiological signal monitoring this manifests primarily as mains power hum of $50 / 60 \mathrm{~Hz}$ although it can also arise from thermal noise (which manifests itself as white noise), shot noise or $1 / \mathrm{f}$ (or pink noise).

In the context of in-home localisation, environmental artifacts generally occur due to deficiencies in the chosen sensing modality. All binary physical interaction sensors and PIR sensors are unable to resolve the identity of the user perturbing a given sensor, hence they are highly susceptible to errors during multiple occupancy. These sensors are also susceptible to misfiring due to changing environmental conditions, leading to incorrect location predictions. These situations can be detected using simple rule-based reasoning [16] and will not be explored further in this paper. All remote interaction binary sensors are susceptible to multiple concurrent sensor firings for a given subject, which must employ artifact removal techniques to resolve these equally valid sensor firings into one true location prediction. Continuous measurements sensors are extremely susceptible to multi-path effects, which refers to reflective, diffractive and scattering interaction of the RF signals with walls and furniture within the environment. Hence, the unpredictable 
variations in RF signals must be compensated for while producing location estimates.

\subsection{Experimental}

Experimental error is such that it can be largely reduced by proper procedure and planning, but it is almost impossible to eliminate completely. Incorrect procedural setup, for example the poor application of electrodes, can create discrepancies in the measured signal, however these errors can often be observed prior to recording, and thus corrected. Motion of the subject can create a large amount of experimental error and is usually the most detrimental. This possible error is far more prominent in the personal health-care environment due to the patient having more freedom of movement.

In relation to the localisation problem, the most significant issues arise when installing the localisation hardware. PIR sensors are line-of-sight technologies, hence if a sensor is placed such that it points out a door, it will detect a subject when he/she is in a different room, leading to an incorrect room prediction. Due to their dependence on multi-path effects, the repeatability of the RF signals at a given location is dependent on the environment configuration. Hence, if the subject reconfigures the environment, by moving furniture or even the localisation hardware, the RF signature at a given location can change in an unpredictable fashion, leading to erroneous location predictions. This is impossible to detect so one must manually ensure that the environment does not significantly change without re-calibrating the localisation system.

\subsection{Physiological}

Physiological artifacts are variations in the desired signal due to other physiological processes in the body. Major contributors of artifacts most commonly detected in physiological measurements are eye movement-related artifacts, cardiac signals and muscle tension signals measured using EMG. Other physiological signals, such as brain signals, do not have sufficient signal strength to cause major complications to the desired measurements.

In terms of location predictions, the entire human body can be considered a physiological artifact. Since the human body has an attenuating effect on RF signals, there will be a different RF signature for different user orientations at a given location. The effect of the human body on RF signals is difficult to mathematically predict in a realistic environment.

Due to the commonly overlapping spectra of the various physiological signals, the artifacts due to undesired biosignals have to be removed in a way that does not involve basic filtering to minimize the amount of lost data. This paper gives a review of a number of the most commonly used algorithms available at present to remove some of the artifacts stated above.

\section{Artifact Removal Techniques}

A review of some of the most commonly implemented techniques to remove the artifacts described in Section 3 is detailed below.

\subsection{Adaptive Filtering}

Adaptive filtering is a commonly used method in biomedical signal processing to remove the unwanted recorded artifacts that contaminate the desired measured physiological signals. An adaptive filter operates by modifying its filter coefficients according to a given optimization algorithm in order to remove undesired noise from a recorded signal. The filter utilizes additional external sensors as a reference for the added noise with the assumption that the added artifact and the desired signal are uncorrelated. Thus as the filter adapts, using the reference to the artifact as input, it progressively removes all artifacts correlated with the reference from the recorded signal. Therefore, the choice of reference is of utmost importance when utilizing the adaptive filter technique.

When removing motion artifacts, the most common artifact references selected are accelerometers [10], however other sources such as optical bend sensors and impedance sensors [15] have also been assessed. Accelerometers are predominantly selected due to their high accuracy level combined with an ease of implementation which is critical for the assisted living domain. Artifacts originating from undesired electrophysiological signals are commonly referenced using recordings from general measurement techniques. The artifact due to the cardiac cycle, often visible in EEG and EMG recordings, are routinely referenced using a ECG measurement across the chest, while artifacts due to the electrical activity of muscles are commonly referenced from the area closest to the recording site where muscle movement occurs, for example around the eye for EEG recordings [17].

The optimization algorithms implemented in the adaptive filter also have significance. Algorithms such as the Least Mean-Squares (LMS) algorithm [20] have a lower computational load than other families of algorithms although as a consequence are not as accurate. Another commonly implemented family of algorithm is the Recursive Least Squares (RLS) algorithm [3] which has a higher computational complexity but with greater accuracy.

In the assisted living environment, the use of adaptive filtering has both advantages and disadvantages. The algorithm is very simple to implement and also is capable of operating on-line and without pre-processing or calibration. However the requirement of a reference signal and thus additional sensors increases the hardware cost and other overheads. The decision of adaptive algorithm is dependent on the computational resources available to the system in operation.

\subsection{Bayesian Filters}

Bayesian filtering is a method of using available noisy observations to probabilistically estimate a given systems' state. Bayesian filters operate on a prediction-correction technique. The time update model is used to describe how the state updates from one time sample to the next. The measurement model is then used to describe how the observed data is related to the internal state of the system. 
This approach overcomes the major limitation of the adaptive filtering technique. The Bayesian filter technique does not require a reference to be used and thus it negates the requirement for additional sensors while, similar to the adaptive filter, the Bayesian filter is capable of operating online.

The Bayesian filter technique is used by a number of different algorithms to remove unwanted artifacts from desired signals, however the Bayesian filter itself cannot be implemented as its algorithm is deemed as impractical.

\subsubsection{Kalman Filter}

The Kalman filter, as described in [32], has two layers of calculations; time update equations and measurement update equations. These equations require a prior knowledge of the process and measurement models, or that the algorithm is calibrated prior to use which could lead to problems in the assisted living domain. One of the main assumptions of the Kalman filter is that the initial uncertainty is Gaussian and that the system dynamics are linear functions of the state [14], however this is not often the case. As most systems are not strictly linear the Kalman filter has also been extended into the non-linear domain through the Extended Kalman Filter (EKF) [32]. The Kalman filter's main advantage over other methods is in the computational efficiency of the algorithm due to its efficient use of matrix operations allowing for longer real-time artifact removal. The Kalman filter and the EKF have been implemented on a number of different signals including PPG [28] and ECG [27], all with promising results.

\subsubsection{Particle Filter}

The Particle filter also implements the Bayesian filter technique but unlike the Kalman filter, does not require the distribution to be unimodal [7]. The Monte Carlo sampling approach is implemented in the Particle filter which allows for multi-modal distributions, while overcoming the mathematical intractability of the Bayes method [12]. This, however, adds further computational complexity to the system causing it to be computationally more expensive then the Kalman filter.

Kalman filters require sensors with high accuracy levels and fast update rates to perform optimally. It is one of the most efficient filtering techniques, which use the Bayesian method, in relation to computation time and memory. However, if accurate sensors are not available, or if there is no detailed model of the process and measurements, then Particle filtering becomes the more viable option. Particle filters do not require a detailed model of the systems and thus are a very flexible tool with a very low implementation overhead [14]. Therefore the choice of algorithm depends, as always, on the sensors and computational power available in the assisted living environment.

\subsubsection{Bayesian Filtering on a Map}

Since RF signals in a home environment are susceptible to multi-path effects, the measurement model is inherently nonlinear and subject to measurement noise, leading to a multimodal distribution of locations corresponding to a given measurement. Furthermore the motion model (referred to as the time update equation in a physiological signal context) is extremely non-linear, since a user can choose to take any path at any point in time. Hence, particle filters are well suited for the Bayesian filter implementation of this system. Furthermore, when a map of the home environment is available, further constraints can be placed on the motion model, allowing particles which are not physically possible to be "killed". For example, particles which propagate through a wall are not permitted but particles which propagate through doorways are permitted. Hence, prediction accuracy can be maximised by using a particle filter to impose constraints on the user's movements [33]. Particle filters, when applied to localisation, can also incorporate readings from multiple sensors into the measurement model, which is referred to sensor fusion. It has been shown that sensor fusion enables the accuracy of the most accurate sensing modality to be achieved at all times [18]. However, as previously stated, particle filters have significant computational overheads which make them unsuitable for online location estimation with high temporal resolution in a home-monitoring context.

Not only do particle filters have a prohibitive computational overhead but they also produce location estimates on the coordinate level, which are not as applicable to a home localisation scenario as symbolic level location. Symbolic location refers to the functional area in which a person resides, such as which room or a subsection of a room they reside. Hence, the computational burden associated with determining coordinate location can be significantly reduced by discretising the home environment into either a grid or a topological representation of symbolic locations. A gridbased approach segments the localisation environment into equally sized discrete cells. Then the Bayes filter prediction and update equations occur on each cell. At each iteration the current location is the cell of highest probability. Thus, arbitrarily shaped distributions can be represented with this technique. Even though a grid-based approach is assumed to be an optimal Bayesian filter when the state-space is truly discrete [26], there still remains the computational complexity of updating the entire grid on every iteration. To maximise computational efficiency, a topological approach can be employed which segments the environment into significant symbolic locations of varying sizes. The motion model is then derived from the connectivity of locations. Topographical approaches have been shown to efficiently represent the localisation environment and perform sufficiently well when the sensors provide imprecise location indicative readings [22].

\subsection{Blind Source Separation (BSS)}

Blind Source Separation (BSS) is based on a family of unsupervised learning algorithms whose objective is to estimate the original sources through estimation of the parameters of the mixing matrix so as to be capable of expressing a reduced representation for the observed data [8]. A BSS algorithm requires no extra external signals, instead it uses the recorded signals and the output of its own algorithm. There are a number of fundamental approaches used to 
separate sources using BSS and these are described by Choi et al. [8]. Independent Component Analysis (ICA) and Canonical Correlation Analysis (CCA), described below, are two of the many algorithms employed in signal analysis that adopt the BSS model. Each algorithm differs in the manner in which it separates the sources. The proper use of these algorithms depends on a priori knowledge of the signal.

\subsubsection{Independent Component Analysis (ICA)}

Independent Component Analysis (ICA) uses the property of independence to separate the signal into its original sources. Although a powerful algorithm, the ICA's major limitations is in its requirement that the independent sources be nonGaussian. ICA can obtain an estimate of the sources iff the sources are non-Gaussian. ICA can, however, operate with a single Gaussian source as this can be determined by the residual remaining after all other sources have been determined. The different algorithms that implement ICA, for example FastICA or the Extended ICA, use different methods to detect independence through non-Gaussianity. These methods include maximizing the magnitude of the kurtosis [30] and maximizing the negentropy [2] of the signal. The ICA algorithms are now a well established method of artifact removal with the choice of method dependent on the problem involved [13] and can also be implemented on single channel recordings [11].

\subsubsection{Canonical Component Analysis (CCA)}

Canonical Correlation Analysis (CCA) is another BSS technique that uses second order statistics (SOS) to generate components. The sources are separated through requiring them to be maximally auto correlated and mutually uncorrelated [9]. This requirement of un-correlation is a weaker condition then the statistical independence sought by the ICA algorithm. CCA, unlike ICA, also takes temporal correlations into account and thus the samples cannot be arranged arbitrarily in time [5]. CCA is also more computationally efficient then ICA as it uses SOS rather than higher order statistics.

A number of authors have reported similar qualitative results from CCA and ICA methods [5] [9], proving that the CCA method could be a favourable method to implement in the assisted living domain where computational resource can be a defining factor.

\subsection{Multiple Binary Sensor Filtering}

As mentioned earlier, binary sensors are susceptible to multiple sensors triggering in different locations at the same time, even during single occupancy. Using rule based reasoning, it can be determined if the multiple firings are possible, by considering the sensor operation and the environment layout [16]. If the firings are impossible, the noisy sensor firing is filtered out. If the sensor firings are all deemed possible then the multiple firings must be aggregated into one location prediction. Multiple sensor triggering generally occurs with remote interaction binary sensors. PIR sensors can have overlapping fields of view. In this scenario, knowledge of the exact areas of sensor overlap must be determined using ray tracing on a map of the environment and then used to construct a lookup table showing sensor coverage. This can be used to determine which area the user must be in to have triggered a particular combination of sensors.

Binary RF sensor firings are more difficult to resolve to location estimates than PIR sensors since RF signals can readily propagate through walls. Furthermore, the attenuative characteristics of each wall are influenced by the type of wall and the angle at which the signal arrives at the wall. Hence, fading and multi-path artifacts make it difficult to mathematically predict the likelihood of detection of an RF signal at a given location. Binary RF localisation generally uses what is referred to as centroid localisation. This algorithm approximates the subject's location to be the mean of the locations of all the receivers which detected the user's RF signal [4]. Hence, RF artifacts can be mitigated by utilising numerous simple RF receivers, with the higher density of receivers leading to higher accuracy. These techniques provide an efficient way of determining location when a high density of simple location sensors is present. However, more reliable localisation is generally possible when several continuous measurement RF sensors are present and the map filtering techniques presented in Section 4.2.3 are employed.

\section{Discussion}

With healthcare moving away from the hospital and towards in home care there are a number of issues that are currently prohibiting a simple and smooth transition. One of the major complications that arise is the increased effect of artifacts on degrading the desired signal. Currently there are a number of techniques available to reduce the effect of these artifacts with each having individual advantages and disadvantages.

There are a number of issues that must be investigated prior to choosing the optimum artifact removal technique for physiological signals. The two most significant are the number of sensors available and the computational cost of the algorithm. In general, as the computational cost of the algorithms increases, so does the accuracy and the robustness. Therefore, for off-line artifact removal, algorithms such as the particle filter would operate to the highest standard. The adaptive filter and CCA algorithms are more suited to online artifact removal due to their lower computational costs and the absence of significant initialisation or modelling overheads. Both the BSS and adaptive filter algorithms can accommodate additional sensors, leading to increased accuracy.

For in-home localisation, the sensing modality employed strongly affects the impact of artifacts on system performance, hence, the most appropriate technique for compensating for the artifacts. In all cases, knowledge of how the sensors behave and interact with each other in the chosen environment must be incorporated into the filtering technique. By exploiting information about the environment configuration, spurious location predictions can be filtered out, maximising performance. 
The selection of which algorithm to implement for use in the assisted living domain is far from complete, however as systems and algorithms continue to improve in computational resources and cost then they will be implemented more readily in non-clinical settings.

\section{Acknowledgements}

This research was supported by the Irish Research Council for Science, Engineering and Technology (IRCSET).

\section{References}

[1] B. Azzerboni et al. "Neural-ICA and wavelet transform for artifacts removal in surface EMG", Neural Networks, 2004. Proceedings. 2004 IEEE International Joint Conference on, volume 4, pp. 3223-3228, (2004)

[2] J. Bell and T.J. Sejnowski. "An information-maximization approach to blind separation and blind deconvolution", Neural Computation, volume 6, pp. 1129-1159, (1995)

[3] T. Benesty, J. Gansler. "New Insights into the RLS Algorithm", EURASIP Journal on Applied Signal Processing, pp. 331-339, (2004)

[4]J. Blumenthal et al. "Weighted centroid localization in zigbee-based sensor networks", Proceedings of IEEE WISP, (2007)

[5] M. Borga and H. Knutsson.. "A canonical correlation approach to blind source separation", Tech Report Linkoping Univ, (2001)

[6] R.T. Brouillette et al. "Nocturnal Pulse Oximetry as an Abbreviated Testing Modality for Pediatric Obstructive Sleep Apnea", Neural Pediatrics, volume 105, pp. 405412, (2000)

[7] Z. Chen. "Bayesian Filtering: From Kalman Filters to Particle Filters, and Beyond", Communications Research Laboratory, McMaster University, (2003)

[8] S Choi et al. "Review blind source separation and independent component analysis: A review", (2004)

[9] W.De Clercq et al. "Canonical correlation analysis applied to remove muscle artifacts from the electroencephalogram", Biomedical Engineering, IEEE Transactions on, volume 53, pp. 2583-2587, (2006)

[10]G. Comtois and Y. Mendelson. "A noise reference input to an adaptive filter algorithm for signal processing in a wearable pulse oximeter", Bioengineering Conference, 2007. NEBC '07, volume 6, pp. 106-107, (2007)

[11]M.E. Davies and C.J. James.. "Source separation using single channel ICA", Signal Processing, volume 87, pp. 1819-1832, (2007)

[12]A. Doucet et al. "Sequential Monte Carlo Methods in Practice (Statistics for Engineering and Information Science)", Springer-Verlag, (2001)

[13]A. Draper et al. "Recognizing faces with pca and ica", Computer vision and image understanding, special issue on face recognition, pp 115--137, (2003)

[14]D. Fox et al. "Bayesian filtering for location estimation", IEEE Pervasive Computing, volume 2, pp. 24-33, (2003)

[15]P.S. Hamilton et al. "Comparison of methods for adaptive removal of motion artifact", Computers in Cardiology, pp 383-386, (2001)
[16]T Hayes et al. "An unobtrusive in-home monitoring system for detection of key motor changes preceding cognitive decline", Proceedings of IEEE EMBC, volume 1, pp. 2480-2483, (2004)

[17]P. He et al. "Removal of ocular artifacts from electroencephalogram by adaptive filtering", Medical and biological engineering and computing, volume 42, pp. 407-412, (2004)

[18]J. Hightower and G. Borriello. "Particle filters for location estimation in ubiquitous computing: A case study", Proceedings of Ubicomp, pp 88-106, (2004)

[19]T.J Huppert et al. "HomER: a review of time-series analysis methods for near-infrared spectroscopy of the brain”, Appl. Opt., volume 48, pp. 280-298, (2009)

[20]D.L. Jones et al. "Adaptive Filtering: LMS Algorithm", Technical report, (2009)

[21]Karlsson et al. "Wireless Monitoring of Heart Rate and Electromyographic Signals using a Smart T-shirt", International Workshop on Wearable Micro and Nanosystems for Personalised Health, (2008)

[22]D. Kelly et al. "A bluetooth-based minimum infrastructure home localisation system", Proceedings of IEEE ISWCS, pp 638-642, (2008)

[23]D. Kelly et al. "Minimal hardware Bluetooth tracking for long-term at-home elder supervision", Proceedings of IEEE EMBC, pp 2136 -- 2140, (2010)

[24]K.W. Kolodziej J. Hjelm "Local Positioning Systems", Taylor \& Francis Group, LLC, (2006)

[25]J.Lundell et al. "Continuous activity monitoring and intelligent contextual prompting to improve medication adherence", Proceedings of IEEE EMBC, (2007)

[26]B. Ristic et al. "Beyond the Kalman Filter: Particle Filters for Tracking Applications", Artech House, (2004)

[27]M. Rohalova et al. "Detection of the EEG Artifacts by the Means of the (Extended) Kalman Filter", Measurement Science Review, (2001)

[28]S. Seyedtabaii and L. Seyedtabaii. "Kalman filter based adaptive reduction of motion artifact from photoplethysmographic signal", World Academy of Science, Engineering and Technology, volume 37, (2008)

[29]L. Sornmo and P. Laguna. "Electrocardiogram (ECG) signal processing", Wiley, (2006)

[30]R. Vigario et al. "Independent component approach to the analysis of EEG and MEG recordings", Biomedical Engineering, IEEE Transactions on, volume 47, pp 589$593,(2000)$

[31]E. Waterhouse. "New horizons in ambulatory electroencephalography", EMB Magazine, volume 22, pp 383-386, (2001)

[32]G. Welsh, G. Bishop. "An Introduction to the Kalman Filter", Tech Report Univ. of North Carolina, Dept. of Computer Science, (2001)

[33]G.V. Zaruba et al. "Indoor location tracking using RSSI readings from a single wi-fi access point", Wireless Networks, volume 13, pp 221-235(2007) 